\title{
Weak coupling expansion of a chiral gauge theory on a lattice in the overlap formulation
}

\author{
Atsushi Yamadaf \\ International Center for Theoretical Physics, \\ Miramare, Trieste, Italy
}

\begin{abstract}
Weak coupling expansion of a chiral gauge theory on a lattice is discussed in the overlap formulation. We analyze the fermion propagator and the fermion-fermion-gauge boson vertex in the one loop level. The chiral properties of the propagator and vertex are correctly preserved without tuning the parameters involved even after the one-loop renormalization, and the ultraviolet divergent parts agree with the continuum theory. Our analysis, together with the existing studies on the vacuum polarization and the gauge boson n-point functions, completes the proof of the renormalizability of this formulation to the one loop level.
\end{abstract}

\footnotetext{
${ }^{\dagger}$ On leave of absence from: Department of Physics, University of Tokyo, Tokyo, 113 Japan.
} 


\section{Introduction.}

The idea of the domain wall fermion [1] may provide a new possibility of regularizing a chiral fermion on a lattice [2, 3, 4. In this approach, a Weyl fermion is simulated by a Dirac fermion in four plus one dimensions. Gauge fields $A_{\mu}(x)$ interacting with the Weyl fermion in four dimensions are extended to four plus one dimensions to be independent of the time coordinate, $A_{\mu}(t, x)=A_{\mu}(x)$ for all $t$. The Dirac fermion in four plus one dimensions is described by two different Hamiltonians $\mathcal{H}_{+}(A)$ for $t<0$ and $\mathcal{H}_{-}(A)$ for $t>0$ where Hamiltonians $\mathcal{H}_{ \pm}(A)$ differ from each other only in the sign of the Dirac mass term. (The subscript ' \pm ' indicates the sign of the mass term.) Because of this step function behavior of the mass term, there arises one zero mode bound to the domain wall (at $t=0$ ), which behaves like a chiral fermion in four dimensions. This domain wall fermion inspired the overlap formulation [3]. To move from the domain wall fermion to the overlap formulation, assume that the four plus one dimensional space is of Minkowskian signature, and consider the path integral representation of the propagator of the domain wall fermion in the presence of the gauge fields,

$$
\left.\int \mathcal{D} \psi \mathcal{D} \bar{\psi} \psi(t, x) \bar{\psi}\left(t^{\prime}, x^{\prime}\right)\right|_{t, t^{\prime}=0} e^{i \int_{-L}^{0} \mathcal{L}_{+}(A) d t+i \int_{0}^{L} \mathcal{L}_{-}(A) d t}
$$

where $\mathcal{L}_{ \pm}(A)$ are the Lagrangians corresponding to $\mathcal{H}_{ \pm}(A)$ and $L$ is the size of the fifth dimension (which is the time coordinate). The Dirac fermion $\psi(t, x)$ at $t=0$ simulates the Weyl fermion and thus eq. (1) simulates the propagator for the Weyl fermion. Taking the limit $L \rightarrow \infty$, eq. (11) will be reduced to,

$$
\left\langle A+\left|T\left\{\psi(t, x) \bar{\psi}\left(t^{\prime}, x^{\prime}\right)\right\}\right|_{t, t^{\prime}=0} \mid A-\right\rangle,
$$

where $|A \pm\rangle$ are the Dirac vacua of the Hamiltonians $\mathcal{H}_{ \pm}(A)$. The overlap formulation directly treats $|A \pm\rangle$ and the form of the propagator (2) without invoking the four plus one dimensional theory. The effective action of a chiral fermion in the presence of the gauge fields (the determinant of the chiral Dirac operator) is expressed by the overlap of the two vacua,

$$
\operatorname{det}\left\{\frac{1}{2}\left(1 \pm \gamma_{5}\right)(\not+i g \not A)\right\} y=e^{-\Gamma(A)} \simeq\langle A+\mid A-\rangle .
$$


The advantage of directly treating the vacuum overlap (3) is that, when the gauge fields exhibit topologically non-trivial configurations, the overlap (3) vanishes [5] (as the chiral determinant should), while if the vacuum overlap is expressed in a similar way to (11) as a limit $L \rightarrow \infty$, it might not vanish due to possible contributions of the excited states of $\mathcal{H}_{ \pm}(A)$.

Based on eq. (3), treating the gauge fields $A_{\mu}(x)$ as slowly varying external fields, it has been in fact confirmed that the vacuum overlap $\langle A+\mid A-\rangle$ correctly reproduces the chiral anomaly [6], the vacuum polarization [7], and gauge boson $n$-point functions [8, 9]. The validity of this formulation has been further examined recently including the dynamics of the gauge fields [10, 11]. The fermion propagator is computed including the self-energy corrections due to the gauge interactions at one-loop level, and has been shown to remain chiral at one loop level without tuning the parameters involved [11]. This analysis suggests the renormalizability of this formulation in the presence of the dynamical fermions and gauge bosons. In this paper, we discuss the weak coupling expansion in the overlap formulation and present the analysis of the propagator and the fermion-fermion-gauge boson vertex at one loop level. The chiral properties of the vertex are correctly preserved as well as those of the propagator, and the ultraviolet divergent parts agree with the continuum theory. Our analysis, together with the studies [7, 9, 11], completes the proof of the renormalizability of this formulation at one loop level. The rest of the paper is organized as follows. In Sec. 2, we briefly review the overlap formulation. After we derive the Hamiltonians $\mathcal{H}_{ \pm}(A)$ and their Dirac vacua $|A \pm\rangle$, we compute the propagator (2) for $A_{\mu}(x)=0$ and analyze its pole. We show that the propagator describes a chiral fermion. In Sec. 3, we first compute the fermion propagator including the one-loop self-energy corrections due to the gauge interactions, and renormalize the propagator. We confirm that the fermion is renormalized preserving the desired chiral structure without tuning the parameters involved. Next, we compute the vertex corrections at one-loop level, and show that the chiral structure and ultraviolet divergences are correctly reproduced. We will give some discussions in Sec. 4. For numerical simulations in the overlap formulation and further applications of this formulation, see Refs. [12] and Refs. [13], respectively. 


\section{Formalism.}

As is discussed in the introduction, the Hamiltonians $\mathcal{H}_{ \pm}(A)$ and their Dirac vacua $|A \pm\rangle$ are the principal quantities in the overlap formulation. First we derive the Hamiltonian $\mathcal{H}_{ \pm}(A)$ from the four plus one dimensional Dirac Lagrangian. Then we express $|A \pm\rangle$ as a perturbation series in the weak coupling expansion.

\section{The overlap Hamiltonians $\mathcal{H}_{ \pm}(A)$ and their Dirac vacua $|A \pm\rangle$.}

The Lagrangians of free Dirac fermions in four plus one dimensions are,

$$
\mathcal{L}_{ \pm}=\bar{\psi}(t, x)\left(i \sum_{A=0}^{5} \Gamma^{A} \partial_{A} \pm T_{c} \Lambda\right) \psi(t, x),
$$

where the mass $\Lambda$ corresponds to the height of the domain wall in the original domain wall fermion in Ref. [1] and $T_{c}$ determines the fermion chirality, as will be seen later. The gamma matrices in four plus one dimensional Minkowskian space satisfy the relations,

$$
\left\{\Gamma^{A}, \Gamma^{B}\right\}=2 \eta_{A B}, \quad \Gamma^{0 \dagger}=\Gamma^{0}, \quad \Gamma^{\mu \dagger}=-\Gamma^{\mu}, \quad(\mu=1, \cdots, 4),
$$

with $\eta_{A B}=(1,-1,-1,-1,-1)$, and they are related to the four dimensional Euclidean gamma matrices 14 as,

$$
\Gamma^{0}=\gamma_{5}^{E}, \quad \Gamma^{\mu}=i \gamma_{\mu}^{E}, \quad\left\{\gamma_{\mu}^{E}, \gamma_{\nu}^{E}\right\}=2 \delta_{\mu \nu}, \quad \gamma_{\mu}^{E \dagger}=\gamma_{\mu}^{E}
$$

(Hereafter we omit the superscript ' $\mathrm{E}^{6}$ of the Euclidean gamma matrices.) The Lagrangians (4) lead to the Hamiltonians,

$$
\mathcal{H}_{ \pm}=\int d^{4} x \psi^{\dagger} \gamma_{5}\left[\sum_{\mu=1}^{4} \gamma_{\mu} \partial_{\mu} \pm T_{c} \Lambda\right] \psi .
$$

These Hamiltonians are discretized as,

$$
\mathcal{H}_{ \pm}=\frac{1}{a} T_{c}( \pm \lambda+4 r) \sum_{x} \bar{\psi}_{n} \psi_{n}-\frac{1}{2 a} \sum_{x}\left[\bar{\psi}_{n}\left(T_{c} r-\gamma_{\mu}\right) \psi_{n+\hat{\mu}}+\bar{\psi}_{n+\hat{\mu}}\left(T_{c} r+\gamma_{\mu}\right) \psi_{n}\right]
$$

by the replacements,

$$
x_{\mu} \rightarrow a n_{\mu}, \quad \psi(x) \rightarrow \psi_{n}, \quad \int d^{4} x \rightarrow \sum_{x}=a^{4} \sum_{n}, \quad \partial_{\mu} \psi_{n} \rightarrow \frac{1}{2 a}\left[\psi_{n+\hat{\mu}}-\psi_{n-\hat{\mu}}\right],
$$


where $a$ is the lattice spacing and we added the Wilson term (multiplied by $T_{c}$ ),

$$
-a^{4} T_{c} \sum \frac{a r}{2} \bar{\psi}_{n} \square \psi_{n}, \square \psi_{n}=\frac{1}{a^{2}} \sum_{\mu}\left[\psi_{n+\hat{\mu}}+\psi_{n-\hat{\mu}}-2 \psi_{n}\right],
$$

with $\Lambda=\lambda / a$. Here $\bar{\psi}=\psi^{\dagger} \gamma_{5}$ because of the relation $\Gamma^{0}=\gamma_{5}$. The gauge interactions are implemented into the Hamiltonians (8), using the link variables $U_{n, n+\hat{\mu}}$, as,

$$
\bar{\psi}_{n} \psi_{n+\hat{\mu}} \rightarrow \bar{\psi}_{n} U_{n, n+\hat{\mu}} \psi_{n+\hat{\mu}}, \quad \bar{\psi}_{n+\hat{\mu}} \psi_{n} \rightarrow \bar{\psi}_{n+\hat{\mu}} U_{n+\hat{\mu}, n} \psi_{n}, \quad U_{n+\hat{\mu}, n}=U_{n, n+\hat{\mu}}^{\dagger}
$$

where, in the weak coupling expansion, the link valuables are expanded in terms of the gauge coupling $g$ with the identifications,

$$
U_{n, n+\hat{\mu}}=e^{i g a A_{\mu}(n)}=1+i g a A_{\mu}(n)+\frac{1}{2 !}\left\{i g a A_{\mu}(n)\right\}^{2}+\cdots .
$$

Here we should note that the gauge fields $A_{\mu}(n)$ describe the gauge interactions in four dimensional Euclidean space and they are completely independent of the time $t$ in the four plus one dimensional sense. (For simplicity, we will consider the Abelian gauge theory.) The Hamiltonians $\mathcal{H}_{ \pm}(A)$ are obtained by inserting eqs. (11) and (12) into eq. (8). For the weak coupling expansion, it is convenient to use momentum representations. Performing the following Fourier transformations,

$$
\psi_{n}=\int_{p} \psi(p) e^{i p a n}, \quad \bar{\psi}_{n}=\int_{q} \bar{\psi}(q) e^{-i q a n}, \quad A_{\mu}(n)=\int_{p} A_{\mu}(p) e^{i p a(n+\hat{\mu} / 2)},
$$

the Hamiltonians (8) with eqs. (11) and (12) are,

$$
\begin{aligned}
& \mathcal{H}_{ \pm}(A)=\int_{p} \psi^{\dagger}(p) H_{ \pm}(p) \psi(p)+\mathcal{V}(A), \\
& H_{ \pm}(p)=\gamma_{5}\left[\sum_{\mu=1}^{4} i \tilde{p}_{\mu} \gamma_{\mu}+T_{c} X_{ \pm}(p)\right], \quad X_{ \pm}(p)= \pm \frac{\lambda}{a}+\frac{a r}{2} \hat{p}^{2} .
\end{aligned}
$$

where $\tilde{p}_{\mu}=(1 / a) \sin \left(p_{\mu} a\right), \hat{p}_{\mu}=(2 / a) \sin \left(p_{\mu} a / 2\right)$ and the momentum integral is over the Brillouin zone $[-\pi / a, \pi / a]$. In eq. (14), the first terms are the free Hamiltonians $\mathcal{H}_{ \pm}(A=0)$. The interaction term $\mathcal{V}$ is treated as perturbations and it is expanded in terms of the gauge coupling $g$ as, $\mathcal{V}=\mathcal{V}_{1}+\mathcal{V}_{2}+\mathcal{V}_{3}+\cdots$, with

$$
\begin{aligned}
& \mathcal{V}_{1}=i g \int_{p, q} \bar{\psi}(p) \sum_{\mu} V_{1 \mu}(p+q) A_{\mu}(p-q) \psi(q), \\
& \mathcal{V}_{2}=\frac{1}{2} a g^{2} \int_{p, s, q} \bar{\psi}(p) \sum_{\mu, \nu} V_{2 \mu}(p+q) \delta_{\mu \nu} A_{\mu}(s) A_{\nu}(p-s-q) \psi(q), \\
& \left.\mathcal{V}_{3}=-\frac{1}{3 !} i a^{2} g^{3} \int_{p, s, t, q} \bar{\psi}(p) \sum_{\mu \nu \tau} \delta_{\mu \nu} \delta_{\mu \tau} V_{3 \mu}(p+q) A_{\mu}(s) A_{\nu}(t) A_{\tau}(p-s-t-q) \psi(q), 16\right)
\end{aligned}
$$


where $V_{1 \mu}(p)=\gamma_{\mu} \cos \left(p_{\mu} a / 2\right)-i r T_{c} \sin \left(p_{\mu} a / 2\right), V_{2 \mu}(p)=T_{c} r \cos \left(p_{\mu} a / 2\right)-i \gamma_{\mu} \sin \left(p_{\mu} a / 2\right)$, and $V_{1 \mu}(p)=V_{3 \mu}(p)$.

To quantize the system, we set the commutation relations,

$$
\left\{\psi_{\alpha m}, \psi_{\beta n}^{\dagger}\right\}=\frac{1}{a^{4}} \delta_{\alpha \beta} \delta_{m n}, \quad\left\{\psi_{\alpha m}, \psi_{\beta n}\right\}=\left\{\psi_{\alpha m}^{\dagger}, \psi_{\beta n}^{\dagger}\right\}=0
$$

which are the equal time commutation relations for a four plus one dimensional Dirac fermion. The operator $\psi(p)$ is expanded in terms of the creation and annihilation operators as,

$$
\psi(p)=\sum_{\sigma}\left[u_{ \pm}(p, \sigma) b_{ \pm}(p, \sigma)+v_{ \pm}(p, \sigma) d_{ \pm}^{\dagger}(p, \sigma)\right]
$$

where $u_{ \pm}$and $v_{ \pm}$are the eigenspinors of the one-particle free Hamiltonians $H_{ \pm}(p)(\sigma$ is the spin index),

$$
\begin{aligned}
H_{ \pm}(p) u_{ \pm}(p, \sigma) & =\omega_{ \pm}(p) u_{ \pm}(p, \sigma), H_{ \pm}(p) v_{ \pm}(p, \sigma)=-\omega_{ \pm}(p) v_{ \pm}(p, \sigma) \\
\omega_{ \pm}(p) & =\sqrt{\tilde{p}^{2}+X_{ \pm}^{2}(p)}
\end{aligned}
$$

which satisfy the orthonormality conditions,

$$
u_{ \pm}(p, \sigma)^{\dagger} u_{ \pm}(p, \tau)=v_{ \pm}(p, \sigma)^{\dagger} v_{ \pm}(p, \tau)=\delta_{\sigma \tau}, \quad u_{ \pm}(p, \sigma)^{\dagger} v_{ \pm}(p, \tau)=0
$$

Their explicit expressions are,

$$
\begin{aligned}
& u_{ \pm}(p, \sigma)=\frac{\omega_{ \pm}+X_{ \pm}-i \sum_{\mu} \tilde{p}_{\mu} \gamma_{\mu} T_{c}}{\sqrt{2 \omega_{ \pm}\left(\omega_{ \pm}+X_{ \pm}\right)}} \chi(\sigma), \\
& v_{ \pm}(p, \sigma)=\frac{\omega_{ \pm}-X_{ \pm}+i \sum_{\mu} \tilde{p}_{\mu} \gamma_{\mu} T_{c}}{\sqrt{2 \omega_{ \pm}\left(\omega_{ \pm}-X_{ \pm}\right)}} \chi(\sigma),
\end{aligned}
$$

where the spinor $\chi(\sigma)$ satisfies $\gamma_{5} T_{c} \chi(\sigma)=\chi(\sigma)$. The creation annihilation operators $\left(b_{+}, d_{+}\right)$satisfy the commutation relations,

$$
\begin{aligned}
& \left\{b_{+}(p, \sigma), b_{+}^{\dagger}(q, \tau)\right\}=\left\{d_{+}(p, \sigma), d_{+}^{\dagger}(q, \tau)\right\}=(2 \pi)^{4} \delta_{\sigma \tau} \delta_{P}^{4}(p-q) \\
& \left\{b_{+}(p, \sigma), d_{+}^{\dagger}(q, \tau)\right\}=\left\{b_{+}(p, \sigma), d_{+}(q, \tau)\right\}=0
\end{aligned}
$$

where $\delta_{P}(p-q)$ is the periodic $\delta$-function on the lattice. (The same relations hold also for $\left.\left(b_{-}, d_{-}\right)\right)$. The two spinor basis are related by,

$$
\begin{aligned}
& u_{-}(p, \sigma)=\cos \beta(p) u_{+}(p, \sigma)-\sin \beta(p) v_{+}(p, \sigma) \\
& v_{-}(p, \sigma)=\sin \beta(p) u_{+}(p, \sigma)+\cos \beta(p) v_{+}(p, \sigma)
\end{aligned}
$$


where $\cos \beta(p)=u_{+}^{\dagger}(p, \sigma) u_{-}(p, \sigma)$ is,

$$
\cos \beta(p)=\frac{1}{\sqrt{2 \omega_{+} 2 \omega_{-}}}\left[\sqrt{\left(\omega_{+}+X_{+}\right)\left(\omega_{-}+X_{-}\right)}+\sqrt{\left(\omega_{+}-X_{+}\right)\left(\omega_{-}-X_{-}\right)}\right] .
$$

Eqs. (23) lead to the Bogoluibov transformation between the two basis $\left(b_{+}, d_{+}\right)$and $\left(b_{-}, d_{-}\right)$,

$$
\begin{aligned}
& b_{-}(p, \sigma)=\cos \beta(p) b_{+}(p, \sigma)-\sin \beta(p) d_{+}^{\dagger}(p, \sigma), \\
& d_{-}^{\dagger}(p, \sigma)=\sin \beta(p) b_{+}(p, \sigma)+\cos \beta(p) d_{+}^{\dagger}(p, \sigma)
\end{aligned}
$$

The commutation relations between $\left(b_{+}, d_{+}\right)$and $\left(b_{-}, d_{-}\right)$are obtained from eqs. (25). For example,

$$
\left\{b_{+}(p, \sigma), b_{-}^{\dagger}(q, \tau)\right\}=\left\{d_{+}(p, \sigma), d_{-}^{\dagger}(q, \tau)\right\}=(2 \pi)^{4} \cos \beta(p) \delta_{\sigma \tau} \delta_{P}^{4}(p-q) .
$$

The Dirac vacua $| \pm\rangle$ for the free Hamiltonians $\mathcal{H}_{ \pm}(A=0)$ are defined as, $b_{+}, d_{+}|+\rangle=0$ and $b_{-}, d_{-}|-\rangle=0$ and their energy eigenvalues are denoted as $E_{ \pm}(0), \mathcal{H}_{ \pm}(A=0)| \pm\rangle=$ $E_{ \pm}(0)| \pm\rangle$. Then for the Dirac vacua $|A \pm\rangle$, the eigenvalue equations,

$$
\mathcal{H}_{ \pm}(A)|A \pm\rangle=E_{ \pm}(A)|A \pm\rangle
$$

are solved in the form of the integral equation using the Dirac vacua $| \pm\rangle$ following the standard time independent perturbation theory, and the results are,

$$
|A \pm\rangle=\alpha_{ \pm}(A)\left[1-G_{ \pm}\left(\mathcal{V}-\Delta E_{ \pm}\right)\right]^{-1}| \pm\rangle
$$

where, $\Delta E_{ \pm}=E_{ \pm}(A)-E_{ \pm}(0)=\langle \pm|\mathcal{V}| A \pm\rangle /\langle \pm \| A \pm\rangle$,

$$
G_{ \pm}=\sum_{n}^{\prime}|n \pm\rangle \frac{1}{E_{ \pm}(0)-E_{ \pm}(n)}\langle n \pm|=\frac{1-| \pm\rangle\langle \pm|}{E_{ \pm}(0)-H_{ \pm}(0)},
$$

and the sum $\sum_{n}^{\prime}$ is over all the excited states $|n \pm\rangle$ of $\mathcal{H}_{ \pm}(0)$ and $E_{ \pm}(n)$ are their energy eigenvalues. The normalization factors $\alpha_{ \pm}(A)$ are determined, up to phases [15, by the normalization conditions of $|A \pm\rangle$ as,

$$
\left|\alpha_{ \pm}(A)\right|^{2}=1-\left\langle A \pm\left|\left[\mathcal{V}-\Delta E_{ \pm}\right] G_{ \pm}^{2}\left[\mathcal{V}-\Delta E_{ \pm}\right]\right| A \pm\right\rangle
$$




\section{Fermion propagator at tree level}

Having presented $\mathcal{H}_{ \pm}(A)$ and $|A \pm\rangle$, we now discuss the fermion propagator. In four plus one dimensions, the propagator is defined by the vacuum expectation value of the T-product,

$$
T\left\{\psi(t, p) \bar{\psi}\left(t^{\prime}, q\right)\right\}=\theta\left(t-t^{\prime}\right) \psi(t, p) \bar{\psi}\left(t^{\prime}, q\right)-\theta\left(t^{\prime}-t\right) \bar{\psi}(t, q) \bar{\psi}(t, p)
$$

Since $\psi(t, p)$ at $t=0$ simulates the Weyl fermion, setting $t, t^{\prime}=0$ and using $\theta(0)=1 / 2$,

$$
\left.T\left\{\psi(t, p) \bar{\psi}\left(t^{\prime}, q\right)\right\}\right|_{t, t^{\prime}=0}=\{\psi(p) \bar{\psi}(q)-\bar{\psi}(q) \psi(p)\} / 2
$$

which we denote as $\Omega(p, q): \Omega(p, q)=\{\psi(p) \bar{\psi}(q)-\bar{\psi}(q) \psi(p)\} / 2$. Then, the fermion propagator is defined by the path integral,

$$
\frac{\int \mathcal{D} \mathcal{A}\langle A+|\Omega(p, q)| A-\rangle e^{-S(A)}}{\int \mathcal{D} \mathcal{A}\langle A+\mid A-\rangle e^{-S(A)}},
$$

where $S(A)$ is the action of the gauge field. We find it convenient for later calculations to decompose $\Omega(p, q)$ in the following 'normal ordered' form (using the Bogoluibov transformation (25)), $\Omega(p, q)=\langle+|\Omega(p, q)|-\rangle+: \Omega(p, q)$;

$$
\begin{aligned}
\langle+|\Omega(p, q)|-\rangle= & (2 \pi)^{4} \delta_{P}^{4}(p-q) \frac{1}{2}\left[S_{+}(p)-S_{-}(p)\right], \\
: \Omega(p, q):= & \frac{1}{\cos \beta(p) \cos \beta(q)} \cdot \sum_{\sigma, \tau}\left[-u_{+}(p, \sigma) \bar{u}_{-}(q, \tau) b_{+}^{\dagger}(q, \tau) b_{-}(p, \sigma)\right. \\
& +u_{+}(p, \sigma) \bar{v}_{+}(q, \tau) b_{-}(p, \sigma) d_{-}(q, \tau)+v_{-}(p, \sigma) \bar{u}_{-}(q, \tau) d_{+}^{\dagger}(p, \sigma) b_{+}^{\dagger}(q, \tau) \\
& \left.+v_{-}(p, \sigma) \bar{v}_{+}(q, \tau) d_{+}^{\dagger}(p, \sigma) d_{-}(q, \tau)\right] .
\end{aligned}
$$

Eq. (34) is the propagator for $A=0$ and the normal ordered product (35) satisfies the relation $\langle+|: \Omega:|-\rangle=0$.

Now we discuss the pole structure of the propagator (34) for the free fermions,

$$
\frac{1}{2}\left[S_{+}(p)-S_{-}(p)\right]=\frac{1}{2 \cos \beta(p)} \sum_{\sigma}\left[u_{+}(p, \sigma) \bar{u}_{-}(p, \sigma)-v_{-}(p, \sigma) \bar{v}_{+}(p, \sigma)\right] .
$$

The spinors $u_{ \pm}, v_{ \pm}$are properly normalized vectors and can not yield divergences in eq. (36) for any value of the momentum $p$. Therefore, the pole of the propagator is given 
by the zero of $\cos \beta(p)$, which occurs only for $\omega_{+}=X_{+}$and $\omega_{-}=-X_{-}$. (The other case, $\omega_{+}=-X_{+}$and $\omega_{-}=X_{-}$, is excluded by the facts $\omega_{+}>\omega_{-}>0$ and $X_{+}>X_{-}$.) The necessary and sufficient condition for the pole of the propagator is $\tilde{p}_{\mu}=0$ and $X_{-}<0$. At the origin of the Brillouin zone $p \simeq 0, \tilde{p}_{\mu}=0$ and $X_{-}=-\lambda / a$, which is the pole of the propagator. At each corner of the Brillouin zone $p_{\mu}= \pm \pi / a+q_{\mu}, \tilde{p}_{\mu}=0$ and $X_{-}=(-\lambda+2 r n) / a$, where $n=1, \cdots, 4$ is the number of momentum components which lie near the corner of the Brillouin zone. Thus, for the range of the parameters $\lambda<2 r$, each corner does not leads to the pole of the propagator. In fact, for $p \simeq 0$, $\omega_{ \pm}(p)=\lambda / a+\mathcal{O}(a), \cos \beta(p)=a \sqrt{p^{2}} / \lambda+\mathcal{O}\left(a^{3}\right)$,

$$
\begin{gathered}
u_{+}(p, \sigma)=\left[1-\frac{a}{2 \lambda} i \not p T_{c}+\mathcal{O}\left(a^{2}\right)\right] \chi(\sigma), \quad u_{-}(p, \sigma)=\frac{1}{\sqrt{p^{2}}}\left[-i \not p T_{c}+\frac{a}{2 \lambda} p^{2}+\mathcal{O}\left(a^{2}\right)\right] \chi(\sigma), \\
\left.v_{-}(p, \sigma)\right)=\left[1+\frac{a}{2 \lambda} i \not p T_{c}+\mathcal{O}\left(a^{2}\right)\right] \chi(\sigma), \quad v_{+}(p, \sigma)=\frac{1}{\sqrt{p^{2}}}\left[i \not p T_{c}+\frac{a}{2 \lambda} p^{2}+\mathcal{O}\left(a^{2}\right)\right] \chi(\sigma),
\end{gathered}
$$

which lead to,

$$
S_{ \pm}(p) \simeq \frac{\lambda}{a} \frac{1}{p^{2}}\left[\frac{1}{2}\left(1+\gamma_{5} T_{c}\right)(\mp i \not p)+\frac{a}{2 \lambda} p^{2} \gamma_{5}\right]
$$

and thus the propagator (36) describes a chiral fermion in this region. At each corner of the Brillouin zone, $p_{\mu} \simeq \pm \pi / a+q_{\mu}$,

$$
\cos \beta(p)=1+\frac{1}{\sqrt{\left(4 n^{2} r^{2}-\lambda^{2}\right)}} \mathcal{O}\left(a^{2} q^{2}\right)
$$

and the propagator takes the following form,

$$
\frac{1}{2}\left[S_{+}(p)-S_{-}(p)\right] \simeq \frac{1}{\sqrt{\left(4 n^{2} r^{2}-\lambda^{2}\right)}+\mathcal{O}\left(a^{2} q^{2}\right)}\left(c_{1}+c_{2} \gamma_{5}\right),
$$

where $c_{1,2}$ are constants. Thus, the chiral non-invariant contributions coming from each corner are suppressed due to the $\sqrt{\left(4 n^{2} r^{2}-\lambda^{2}\right)}$ mass.

\section{The weak coupling expansion.}

Next consider the effects of the gauge interactions at one loop level in the weak coupling expansion. 


\section{Fermion propagator}

First consider the fermion propagator. To obtain fermion propagator at one loop level, the expression (33) should be expanded up to the order $g^{2}$. Inserting the decomposition of $\Omega(p, q)$ into eq. (33), eq. (34) yields the propagator at tree level and all the quantum corrections arise from $\langle A+|: \Omega:| A-\rangle$. Expanding $|A \pm\rangle$ in the perturbation series,

$$
|A \pm\rangle=\alpha_{ \pm}(A)\left[| \pm\rangle+G_{ \pm} \mathcal{V}| \pm\rangle+G_{ \pm}\left\{\mathcal{V}-\Delta E_{ \pm}\right\} G_{ \pm} \mathcal{V}| \pm\rangle+\cdots\right]
$$

the quantum correction $\langle A+|: \Omega:| A-\rangle$, up to the order $g^{2}$, is,

$$
\begin{aligned}
& \langle A+|: \Omega:| A-\rangle=\left\langle+\left|: \Omega: G_{-} \mathcal{V}_{2}\right|-\right\rangle+\left\langle+\left|\mathcal{V}_{2} G_{+}: \Omega:\right|-\right\rangle+\left\langle+\left|\mathcal{V}_{1} G_{+} \mathcal{V}_{1} G_{+}: \Omega:\right|-\right\rangle \\
& +\left\langle+\left|\mathcal{V}_{1} G_{+}: \Omega: G_{-} \mathcal{V}_{1}\right|-\right\rangle+\left\langle+\left|: \Omega: G_{-} \mathcal{V}_{1} G_{-} \mathcal{V}_{1}\right|-\right\rangle .
\end{aligned}
$$

(The terms containing $\Delta E_{ \pm}$are neglected since they are of order $\mathcal{O}\left(g^{2}\right)$.) These terms are evaluated by rewriting the fermion operators in $\mathcal{V}_{1}$ and $\mathcal{V}_{2}$ in terms of the creation and annihilation operators defined in eq. (18) and using the commutation relations. As an example, we evaluate the third term in eq (42). Using eq. (29),

$$
\begin{aligned}
\left\langle+\left|\mathcal{V}_{1} G_{+} \mathcal{V}_{1} G_{+}: \Omega:\right|-\right\rangle= & \sum_{n_{A}, n_{B}}{ }^{\prime}\left\langle+\left|\mathcal{V}_{1}\right| n_{A}+\right\rangle \frac{1}{E_{+}(0)-E_{+}\left(n_{A}\right)}\left\langle n_{A}+\left|\mathcal{V}_{1}\right| n_{B}+\right\rangle \\
& \cdot \frac{1}{E_{+}(0)-E_{+}\left(n_{B}\right)}\left\langle n_{B}+|: \Omega:|-\right\rangle .
\end{aligned}
$$

Here, $\left|n_{A}+\right\rangle$ should be two particle states,

$$
\left|n_{A}+\right\rangle=\left|1_{A} \overline{1}_{A}+\right\rangle=b_{+}^{\dagger}\left(1_{A}\right) d_{+}^{\dagger}\left(\overline{1}_{A}\right)|+\rangle,
$$

where we use the abbreviation $b_{+}^{\dagger}\left(1_{A}\right)=b_{+}^{\dagger}\left(p_{1 A}, \sigma_{1 A}\right)$ e.t.c. For $\left|n_{B}+\right\rangle$, both two and four particle states contribute. We only consider four particle states in our example,

$$
\left|n_{B}+\right\rangle=\left|1_{B} \overline{1}_{B} 2_{B} \overline{2}_{B}+\right\rangle=b_{+}^{\dagger}\left(2_{B}\right) d_{+}^{\dagger}\left(\overline{2}_{B}\right) b_{+}^{\dagger}\left(1_{B}\right) d_{+}^{\dagger}\left(\overline{1}_{B}\right)|+\rangle .
$$

The matrix elements of $\mathcal{V}_{1}$ between relevant states are given by,

$$
\begin{aligned}
\left\langle+\left|\mathcal{V}_{1}\right| 1_{A} \overline{1}_{A}+\right\rangle= & \bar{v}_{+}\left(\overline{1}_{A}\right) \Gamma_{1}\left(\overline{1}_{A}, 1_{A}\right) \bar{u}_{+}\left(1_{A}\right), \\
\left\langle 1_{A} \overline{1}_{A}+\left|\mathcal{V}_{1}\right| 1_{B} \overline{1}_{B} 2_{B} \overline{2}_{B}+\right\rangle= & \left\langle+\left|\mathcal{V}_{1}\right| 2_{B} \overline{2}_{B}+\right\rangle\left\langle 1_{A} \overline{1}_{A}+\mid 1_{B} \overline{1}_{B}+\right\rangle-\left(1_{B} \leftrightarrow 2_{B}\right)-\left(\overline{1}_{B} \leftrightarrow \overline{2}_{B}\right) \\
& +\left(1_{B} \overline{1}_{B} \leftrightarrow 2_{B} \overline{2}_{B}\right),
\end{aligned}
$$


where,

$$
\begin{aligned}
& \Gamma_{1}(p, q)=i g \sum_{\mu} V_{1 \mu}(p+q) A_{\mu}(p-q), \quad\left\langle 1_{A} \overline{1}_{A}+\mid 1_{B} \overline{1}_{B}+\right\rangle=\delta\left(1_{A}, 1_{B}\right) \delta\left(\overline{1}_{A}, \overline{1}_{B}\right), \\
& \delta\left(1_{A}, 1_{B}\right)=(2 \pi)^{4} \delta_{\sigma_{1_{A}} \sigma_{1_{B}}} \delta_{P}^{4}\left(p_{1_{A}}-p_{1_{B}}\right) .
\end{aligned}
$$

Inserting eqs. (46) into eq. (43),

$$
\begin{aligned}
\langle+| \mathcal{V}_{1} G_{+} \mathcal{V}_{1} G_{+}= & \frac{1}{2 ! 2 !} \int_{1_{B} \overline{1}_{B} 2_{B} \overline{2}_{B}}\left[\left\{\frac{-1}{\omega_{+}\left(1_{B}\right)+\omega_{+}\left(\overline{1}_{B}\right)}\right\}\left\langle+\left|\mathcal{V}_{1}\right| 1_{B} \overline{1}_{B}+\right\rangle\right. \\
& \cdot\left\{\frac{-1}{\omega_{+}\left(1_{B}\right)+\omega_{+}\left(\overline{1}_{B}\right)+\omega_{+}\left(2_{B}\right)+\omega_{+}\left(\overline{2}_{B}\right)}\right\}\left\langle+\left|\mathcal{V}_{1}\right| 2_{B} \overline{2}_{B}+\right\rangle \\
& \left.-\left(1_{B} \leftrightarrow 2_{B}\right)-\left(\overline{1}_{B} \leftrightarrow \overline{2}_{B}\right)+\left(1_{B} \overline{1}_{B} \leftrightarrow 2_{B} \overline{2}_{B}\right)\right] \\
& \cdot\left\langle 1_{B} \overline{1}_{B} 2_{B} \overline{2}_{B}+\right| .
\end{aligned}
$$

The normal ordered part is evaluated using eq. (35) as,

$$
\begin{aligned}
\left\langle 1_{B} \overline{1}_{B} 2_{B} \overline{2}_{B}+|: \Omega:|-\right\rangle= & -\sum_{\sigma, \tau} \frac{1}{c_{\beta}(p) c_{\beta}(q)} v_{-}(p, \sigma) \bar{u}_{-}(q, \tau) \delta\left(2_{B}, q \tau\right) \delta\left(\overline{2}_{B}, p \sigma\right)\left\langle 1_{B} \overline{1}_{B}+\mid-\right\rangle \\
& +\left(1_{B} \leftrightarrow 2_{B}\right)+\left(\overline{1}_{B} \leftrightarrow \overline{2}_{B}\right)-\left(1_{B} \overline{1}_{B} \leftrightarrow 2_{B} \overline{2}_{B}\right) \\
& \left\langle 1_{B} \overline{1}_{B}+\mid-\right\rangle=\frac{s_{\beta}\left(1_{B}\right)}{c_{\beta}\left(1_{B}\right)} \delta\left(1_{B}, \overline{1}_{B}\right) .
\end{aligned}
$$

Combining eqs. (48) and (49),

$$
\begin{aligned}
& \left\langle+\left|\mathcal{V}_{1} G_{+} \mathcal{V}_{1} G_{+}: \Omega:\right|-\right\rangle=\frac{2 ! 2 !}{2 ! 2 !} \int_{1_{B} \overline{1}_{B} 2_{B} \overline{2}_{B}}\left\{\frac{1}{\omega_{+}\left(1_{B}\right)+\omega_{+}\left(\overline{2}_{B}\right)}\right\}\left\langle+\left|\mathcal{V}_{1}\right| 1_{B} \overline{2}_{B}+\right\rangle \\
& \cdot\left\{\frac{1}{\omega_{+}\left(1_{B}\right)+\omega_{+}\left(\overline{2}_{B}\right)+\omega_{+}\left(2_{B}\right)+\omega_{+}\left(\overline{1}_{B}\right)}\right\}\left\langle+\left|\mathcal{V}_{1}\right| 2_{B} \overline{1}_{B}+\right\rangle \frac{1}{c_{\beta}(p) c_{\beta}(q)} v_{-}(p, \sigma) \bar{u}_{-}(q, \tau) \\
& \cdot \delta\left(2_{B}, q \tau\right) \delta\left(\overline{2}_{B}, p \sigma\right)\left\langle 1_{B} \overline{1}_{B}+\mid-\right\rangle+(1 \text { more term }), \\
& =S_{-}(p) \int_{p_{1}}\left\{\frac{1}{\omega_{+}\left(1_{B}\right)+\omega_{+}(p)}\right\}\left\{\frac{1}{\omega_{+}(p)+\omega_{+}(q)+2 \omega_{+}\left(1_{B}\right)}\right\} \Gamma_{1}\left(p, 1_{B}\right) \\
& \cdot T_{+}\left(1_{B}\right) \Gamma_{1}\left(1_{B}, q\right) S_{+}(q)+(1 \text { more term }),
\end{aligned}
$$

where,

$$
T_{+}(p)=\sum_{\sigma} \frac{s_{\beta}(p)}{c_{\beta}(p)} u_{+}(p, \sigma) \bar{v}_{+}(p, \sigma),
$$

and we only considered the one-particle irreducible contributions. (The one-particle reducible contributions vanish because there is no tadpole contribution for the gauge fields. 
Namely, the terms containing the combination, for example, $\left\langle+\left|\mathcal{V}_{1}\right| 1_{B} \overline{1}_{B}+\right\rangle\left\langle 1_{B} \overline{1}_{B}+\mid-\right\rangle$, vanish after the momentum integration because of the discrete lattice symmetry.)

Now, $\Gamma_{1}(p, q)$ is rewritten in terms of the gauge fields by eq. (47), and the path integral over the gauge fields at the stage of eq. (33) leads to the following propagator,

$$
\left\langle A_{\mu}(p) A_{\nu}(q)\right\rangle=(2 \pi)^{4} \delta_{P}^{4}(p+q) e^{-i(p+q)_{\nu} a / 2} D_{\mu \nu}(p), \quad D_{\mu \nu}(p)=\frac{1}{\hat{p}^{2}}\left(\delta_{\mu \nu}-(1-\xi) \frac{\hat{p}_{\mu} \hat{p}_{\nu}}{\hat{p}^{2}}\right),
$$

for the bilinear of the gauge fields $A_{\mu}(p) A_{\nu}(q)$. Summing up all the eight terms, the contributions of the fourth term in eq. (42) are expressed as $-(2 \pi)^{4} \delta(p-q) S_{-}(p) a \Sigma(p) S_{+}(p)$, where

$$
\Sigma(p)=\frac{1}{a} \bar{g}^{2} \sum_{\mu, \nu} \int_{k}\left[\frac{1}{\omega_{ \pm}(p)+\omega_{ \pm}(k)}\right]^{2} V_{1 \mu}(p+k) T_{+}(k) V_{1 \nu}(p+k) D_{\mu \nu}(p-k) .
$$

Here we explicitly factor out the lattice spacing $a$ so that $\Sigma(p)$ has the correct dimension (one) of the self-energy for fermions. As is seen from above, this term is described by the Feynman diagram Fig. 1. (a).

Next we compute eq. (53) in the limit $a \rightarrow 0$. We will follow the strategy developed in Ref. [16, 17, 18]. First to see the divergences associated with the limits $a \rightarrow 0$, we factor out the $a$ dependence from the loop momentum integration by a rescaling of the loop momentum $k \rightarrow \bar{k}=k a$. Then the limit $a \rightarrow 0$ produces the linear and logarithmic divergences. Here, the logarithmic divergence is associated with the infrared divergence of the $\bar{k}$ integration in the limit $a p \rightarrow 0$. Such an infrared divergence takes place only in the region $\bar{k} \simeq 0$ because of the pole structure of the propagators (38) and (52). To evaluate these divergent terms, we expand the integrand with respect to the external momentum $p$ around $p \simeq 0$. Then, the zero and first terms of the Taylor expansion contain linear and logarithmic divergences, respectively. As a consequence of the expansion around $p \simeq 0$, the $\bar{k}$ integration also develops infrared divergences associated with $p \rightarrow 0$ (not $a \rightarrow 0$ ), so we regularize infrared divergences by dimensional regularization from the beginning. (Another way of evaluating eq. (53) in the limit $a \rightarrow 0$ is to divide the integration region into two pieces, which is described in Ref. [18.)

Starting from the expression (53), first we analytically continue the space-time dimension $D$ larger than 4 to regularize infrared divergences and introduce an arbitrary 
parameter $\kappa$ of mass dimension one to keep the dimensions of the vertices unchanged [19],

$$
\int_{-\pi / a}^{\pi / a}\left(\frac{d k}{2 \pi}\right)^{4} \rightarrow \kappa^{2 \varepsilon} \int_{-\pi / a}^{\pi / a}\left(\frac{d k}{2 \pi}\right)^{D}, \quad \varepsilon=\frac{4-D}{2},
$$

Then rescaling the loop momentum $k \rightarrow \bar{k}=k a$,

$$
\begin{aligned}
\Sigma(p)= & \frac{1}{a} g^{2} \sum_{\mu, \nu}(\kappa a)^{2 \varepsilon} \int_{-\pi}^{\pi}\left(\frac{d \bar{k}}{2 \pi}\right)^{D}\left[\frac{1}{\tilde{\omega}_{+}(\bar{k})+\tilde{\omega}_{+}(a p)}\right]^{2} \tilde{V}_{1 \mu}(a p+\bar{k}) \tilde{T}_{+}(\bar{k}) \\
& \cdot \tilde{V}_{1 \nu}(\bar{k}+a p) \tilde{D}_{\mu \nu}(a p-\bar{k}),
\end{aligned}
$$

where the rescaling of each quantity is,

$$
\omega_{+}(k)=\frac{1}{a} \tilde{\omega}_{+}(\bar{k}), \quad V_{1 \mu}(k)=\tilde{V}_{1 \mu}(\bar{k}), \quad T_{+}(k)=\tilde{T}_{+}(\bar{k}), \quad D_{\mu \nu}(k)=a^{2} \tilde{D}_{\mu \nu}(\bar{k}) .
$$

Next, expanding eq. (55) with respect to the external momentum $p$, the momentum integral is finite for the zero term, and gives rise to,

$$
\Sigma(0)=\frac{1}{a}\left[\sigma_{1}(\lambda, r, \xi)+\sigma_{2}(\lambda, r, \xi) \gamma_{5}\right],
$$

where $\sigma_{1,2}(\lambda, r, \xi)$ are the constants. For the first term,

$$
\left.\sum_{\mu} p_{\mu} \frac{\partial \Sigma(p)}{\partial p_{\mu}}\right|_{p=0}
$$

only the derivative of $\tilde{D}_{\mu \nu}(a p-\bar{k})$ leads to the integral which gives rise to the logarithmic (infrared) divergence, and the derivative of the other terms yields, after the integration, finite terms in the limit $\varepsilon, a \rightarrow 0$. Computing the integral, we obtain,

$$
\begin{aligned}
\left.\sum_{\mu} p_{\mu} \frac{\partial \Sigma(p)}{\partial p_{\mu}}\right|_{p=0} \rightarrow & \frac{1}{\lambda} \frac{1}{4} \frac{\bar{g}^{2}}{16 \pi^{2}}\{1-(1-\xi)\}\left\{\frac{1}{\varepsilon}+\log \left(\kappa^{2} a^{2}\right)\right\} \\
& \cdot \frac{1}{2}\left(1-\gamma_{5} T_{c}\right) \text { ipp }+ \text { finite term. }
\end{aligned}
$$

The remaining part,

$$
\Sigma(p)-\Sigma(0)-\left.\sum_{\mu} p_{\mu} \frac{\partial \Sigma(p)}{\partial p_{\mu}}\right|_{p=0}
$$

is ultraviolet finite for $a \rightarrow 0$, but is infrared divergent for $\varepsilon \rightarrow 0$. In fact, because of the subtraction of the first two terms of the Taylor expansion, eq. (60) would have been of 
order $a$ if there were no infrared divergences at $a p \rightarrow 0$. Dividing the integration region into parts where the integral is regular in the limit $a p \rightarrow 0$ and parts where the integral exhibits an infrared divergence, the contribution of the former is irrelevant, while that of the latter is finite for $a \rightarrow 0$. Because of the structure of the poles of the propagators (38) and (52) such an infrared divergence can occur only at $\bar{k} \simeq 0$ in the limit $a p \rightarrow 0$, and the contribution of this region is evaluated by expanding the integrand around $\bar{k} \simeq 0$ (and $a p \simeq 0$ ). The result of the calculation is,

$$
\begin{gathered}
\Sigma(p)-\Sigma(0)-\left.\sum_{\mu} p_{\mu} \frac{\partial \Sigma(p)}{\partial p_{\mu}}\right|_{p=0} \\
\rightarrow \quad-\frac{1}{\lambda} \frac{1}{4} \frac{g^{2}}{16 \pi^{2}}\{1-(1-\xi)\}\left\{\frac{1}{\varepsilon}+\log \left(\kappa^{2} / p^{2}\right)\right\} \frac{1}{2}\left(1-\gamma_{5} T_{c}\right) i \not p .
\end{gathered}
$$

Summing up eqs. (57), (59) and (61), we obtain,

$$
\begin{aligned}
\Sigma(p)= & \frac{1}{a}\left[\sigma_{1}(\lambda, r, \xi)+\sigma_{2}(\lambda, r, \xi) \gamma_{5}\right]+\frac{1}{\lambda} \frac{1}{4} \frac{g^{2}}{16 \pi^{2}}\{1-(1-\xi)\} \log \left(p^{2} a^{2}\right) \\
& \cdot \frac{1}{2}\left(1-\gamma_{5} T_{c}\right) i \not p+(\text { finite terms }) .
\end{aligned}
$$

The infrared divergences at $\varepsilon \rightarrow 0$ are cancelled in eq. (62). The contribution to the tree level propagator is given by,

$$
\begin{aligned}
& -S_{-}(p) a \Sigma(p) S_{+}(p)=-\frac{\lambda}{a} \frac{1}{p^{2}}\left[\frac{1}{2}\left(1+\gamma_{5} T_{c}\right)(+i \not p)+\frac{a}{2 \lambda} p^{2} \gamma_{5}\right] a \\
& \cdot\left[\frac{1}{a}\left\{\sigma_{1}+\sigma_{2} \gamma_{5}\right\}+\frac{1}{\lambda} \frac{1}{4} \frac{g^{2}}{16 \pi^{2}}\{1-(1-\xi)\} \log \left(p^{2} a^{2}\right) \frac{1}{2}\left(1-\gamma_{5} T_{c}\right) i \not p+(\text { finite terms })\right] \\
& \cdot \frac{\lambda}{a} \frac{1}{p^{2}}\left[\frac{1}{2}\left(1+\gamma_{5} T_{c}\right)(-i \not p)+\frac{a}{2 \lambda} p^{2} \gamma_{5}\right] \\
\rightarrow \quad & {\left[\sigma_{1} T_{c}+\frac{1}{\lambda} \frac{1}{4} \frac{g^{2}}{16 \pi^{2}}\{1-(1-\xi)\} \log \left(p^{2} a^{2}\right)+\text { finite terms }+\mathcal{O}(a)\right] } \\
& \cdot \frac{\lambda}{a} \frac{1}{2}\left(1+\gamma_{5} T_{c}\right) \frac{-i \not p}{p^{2}} .
\end{aligned}
$$

From this expression, we can see that only an ultraviolet divergence of $\Sigma(p)$ more severe than quadratic can invalidate the chiral property of the regularized fermion, and the logarithmically divergent term in eq. (62) directly appears in the wave function renormalization factor as a logarithmic divergence. 
If we take two particle contributions for $\left|n_{B}+\right\rangle$ in eq. (45), we obtain, instead of eq. (53),

$$
\begin{aligned}
\Sigma(p)= & -\frac{1}{a} g^{2} \frac{1}{2 \omega_{+}(p)} \sum_{\mu, \nu} \int_{k} \frac{1}{\omega_{+}(p)+\omega_{+}(k)} V_{1 \mu}(p+k) \\
& \cdot \sum_{s}\left[u_{+}(k, s) \bar{u}_{+}(k, s)-v_{+}(k, s) \bar{v}_{+}(k, s)\right] V_{1 \nu}(p+k) D_{\mu \nu}(p-k) .
\end{aligned}
$$

This term does not contain logarithmic divergences since there appears no infrared divergence for the first term of the Taylor expansion after the rescaling and expansion.

The other terms in eq. (42) are evaluated in the same way. (The results are given in Ref. [11]). The first two terms are expressed by the Feynman diagram Fig. 1. (b), and do not lead to the logarithmic divergence at the level of eq. (63), while the fourth and fifth terms correspond to the Feynman diagram Fig. 1. (a), and yield the logarithmic divergences. Summing up all the contributions as well as the propagator at tree level, the propagator at one loop level is,

$$
\frac{\lambda}{a} \frac{1}{2}\left(1+\gamma_{5} T_{c}\right) \frac{-i \not p}{p^{2}}\left[1+\frac{\bar{g}^{2}}{16 \pi^{2}}\{1-(1-\xi)\}\left(\frac{1}{4}+\frac{1}{2}+\frac{1}{4}\right)\left(\log a^{2} p^{2}+\text { finite terms }\right)\right],
$$

where the contributions of the third and fourth and fifth terms to the logarithmically divergent term are 1/4,1/2 and 1/4, respectively. From this expression, we see that the chirality of the regularized fermion is properly preserved and the wave function renormalization factor is,

$$
Z_{2}=1+\frac{g^{2}}{16 \pi^{2}}[1-(1-\xi)]\left(\log a^{2} \mu^{2}+\text { const }\right),
$$

where $\mu$ is the renormalization scale. The divergent part of the wave function renormalization factor agrees with that of the continuum theory.

\section{Vertex correction}

Next we consider fermion-fermion-gauge boson vertex. The three point function is given by,

$$
\frac{\int \mathcal{D} \mathcal{A}\langle A+|\Omega(p, q)| A-\rangle A_{\mu}(t) e^{-S(A)}}{\int \mathcal{D} \mathcal{A}\langle A+\mid A-\rangle e^{-S(A)}} .
$$


To obtain the vertex up to the one loop level, eq. (67) should be expanded up to the order $g^{3}$. The procedure of the calculation is same to the case of the propagator. Inserting the decomposition of $\Omega(p, q)$ into eq. (67), the first part $\langle+|\Omega(p, q)|-\rangle$ vanishes after the path integration over the gauge fields. The normal ordered part $\langle A+|: \Omega(p, q):| A-\rangle$ is evaluated up to the order $g^{3}$ by expanding $|A \pm\rangle$ with respect to the gauge coupling $g$ in eq. (28). Up to this order,

$$
\begin{aligned}
& \langle A+|: \Omega:| A-\rangle=\alpha_{+}^{*}(A) \alpha_{-}(A)\left\{\left\langle+\left|\mathcal{V} G_{+}: \Omega:\right|-\right\rangle+\left\langle+\left|: \Omega: G_{-} \mathcal{V}\right|-\right\rangle+\left\langle+\left|\mathcal{V} G_{+} \mathcal{V} G_{+}: \Omega:\right|-\right\rangle\right. \\
& +\left\langle+\left|\mathcal{V} G_{+}: \Omega: G_{-} \mathcal{V}\right|-\right\rangle+\left\langle+\left|: \Omega: G_{-} \mathcal{V} G_{-} \mathcal{V}\right|-\right\rangle+\left\langle+\left|\mathcal{V} G_{+} \mathcal{V} G_{+} \mathcal{V} G_{+}: \Omega:\right|-\right\rangle \\
& +\left\langle+\left|\mathcal{V} G_{+} \mathcal{V} G_{+}: \Omega: G_{-} \mathcal{V}\right|-\right\rangle+\left\langle+\left|\mathcal{V} G_{+}: \Omega: G_{-} \mathcal{V} G_{-} \mathcal{V}\right|-\right\rangle+\left\langle+\left|: \Omega: G_{-} \mathcal{V} G_{-} \mathcal{V} G_{-} \mathcal{V}\right|-\right\rangle \\
& \left.-\Delta E_{+}\left\langle+\left|\mathcal{V} G_{+}^{2}: \Omega:\right|-\right\rangle-\Delta E_{-}\left\langle+\left|: \Omega: G_{-}^{2} \mathcal{V}\right|-\right\rangle\right\}
\end{aligned}
$$

The first two terms $\left\langle+\left|\mathcal{V} G_{+}: \Omega:\right|-\right\rangle$ and $\left\langle+\left|: \Omega: G_{-} \mathcal{V}\right|-\right\rangle$ with $\mathcal{V}=\mathcal{V}_{1}$ lead to the vertex at tree level after the path integration over $A_{\mu}$,

$$
\begin{gathered}
(2 \pi)^{4} \delta_{P}(p+t-q) \sum_{\rho} D_{\mu \rho}(t) e^{-\frac{i}{2}(p+t-q) \rho}\left\{S_{-}(p) \frac{a}{\lambda} \Gamma_{\rho}(p, q) S_{+}(q)+S_{+}(p) \frac{a}{\lambda} \Gamma_{\rho}(p, q) S_{-}(q)\right\}, \\
\Gamma_{\rho}(p, q) \rightarrow \frac{1}{2} i g \frac{1}{2}\left(1-\gamma_{5} T_{c}\right) \gamma_{\rho}, \quad(a \rightarrow 0) .
\end{gathered}
$$

For the radiative corrections we only need to evaluate one-particle irreducible contributions. The vertex corrections exhibit logarithmic divergences, which associate with infrared divergences of the integral after the rescaling of the loop momentum in our strategy. Therefore, in this case, we essentially look for terms which have enough poles, or equivalently $\cos \beta(p)^{-1}$ factors, to exhibit infrared divergences. Such terms are obtained only from the interaction $\mathcal{V}_{1}$, and the contributions of $\mathcal{V}_{2}$ and $\mathcal{V}_{3}$ yield only finite contributions.

For example, we evaluate a term containing $\mathcal{V}_{2},\left\langle+\left|G_{+} \mathcal{V}_{1} G_{+} \mathcal{V}_{2}: \Omega:\right|-\right\rangle$. This term leads to the expression,

$$
\begin{aligned}
& \left\langle+\left|G_{+} \mathcal{V}_{1} G_{+} \mathcal{V}_{2}: \Omega:\right|-\right\rangle \rightarrow \frac{2 ! 2 !}{2 ! 2 !} \int_{2_{B}}\left\{\frac{1}{\omega_{+}(q)+\omega_{+}\left(2_{B}\right)}\right\}\left\{\frac{1}{\omega_{+}(q)+\omega_{+}(p)+2 \omega_{+}\left(2_{B}\right)}\right\} \\
& \cdot S_{-}(p) \Gamma_{2}\left(p, 2_{B}\right) T_{+}\left(2_{B}\right) \Gamma_{1}\left(2_{B}, q\right) S_{+}(q)+(1 \text { more term }),
\end{aligned}
$$

where,

$$
\Gamma_{1}(p, q)=i g \sum_{\mu} V_{1 \mu}(p+q) A_{\mu}(p-q)
$$




$$
\Gamma_{2}(p, q)=\frac{1}{2} a g^{2} \int_{s} \sum_{\mu, \nu} V_{2 \mu}(p+q) \delta_{\mu \nu} A_{\mu}(s) A_{\nu}(p-s-q),
$$

and we have considered only the four particle contributions for the evaluation of the second $G_{+}$between $\mathcal{V}_{1}$ and $\mathcal{V}_{2}$. (The two particle contributions for second $G_{+}$do not give rise to enough pole factors to exhibit infrared divergences.) After the path integration over the gauge fields, the first term in eq. (70) yields the following one-particle irreducible contribution,

$$
\begin{aligned}
& (2 \pi)^{4} \delta_{P}(p+t-q) \sum_{\rho} D_{\mu \rho}(t) e^{-\frac{i}{2}(p+t-q)_{\rho} a}\left\{S_{-}(p) \frac{a}{\lambda} \Gamma_{\rho}(p, q) S_{+}(q)\right\}, \\
& \Gamma_{\rho}(p, q)=\frac{\lambda}{a} i g \frac{1}{2} a g^{2} \int_{k}\left\{\frac{1}{\omega_{+}(p)+\omega_{+}(k)}\right\}\left\{\frac{1}{\omega_{+}(p)+\omega_{+}(q)+2 \omega_{+}(k)}\right\} \\
& \cdot \sum_{\nu} V_{1 \nu}(p+k) T_{+}(k) V_{2 \rho}(k+q) D_{\nu \rho}(p-k) .
\end{aligned}
$$

This contribution is described by the Feynman diagram in Figs. 2. (a). Rescaling the momentum variable $k \rightarrow \bar{k}=a k$, we only need to evaluate the zero term of the Taylor expansion with respect to the external momenta $p$ and $q$ to compute possible divergent terms in the limit $a \rightarrow 0$, which is,

$$
\Gamma_{\rho}(p=0, q=0)=\frac{\lambda}{a} i g \frac{1}{4} a g^{2} \int_{\bar{k}}\left\{\frac{1}{\lambda+\tilde{\omega}_{+}(\bar{k})}\right\}^{2} \sum_{\nu} \tilde{V}_{1 \nu}(\bar{k}) \tilde{T}_{+}(\bar{k}) \tilde{V}_{2 \rho}(\bar{k}) \tilde{D}_{\nu \rho}(-\bar{k}) .
$$

The integral over $\bar{k}$ is (infrared) finite because of the structure of the interaction vertices, and thus this term only yields the finite contributions. The contributions of the terms $\left\langle+\left|\mathcal{V}_{3} G_{+}: \Omega:\right|-\right\rangle$ and $\left\langle+\left|: \Omega: G_{-} \mathcal{V}_{3}\right|-\right\rangle$ are described by the Feynman diagram Fig. 2. (b) and yield only finite corrections to the vertex. In this way, the interactions $\mathcal{V}_{2}$ and $\mathcal{V}_{3}$ lead only to the finite terms (contact terms).

The logarithmically divergent terms are obtained from the following terms in eq. (68),

$$
\begin{aligned}
& \left\langle+\left|\mathcal{V} G_{+} \mathcal{V} G_{+} \mathcal{V} G_{+}: \Omega:\right|-\right\rangle+\left\langle+\left|\mathcal{V} G_{+} \mathcal{V} G_{+}: \Omega: G_{-} \mathcal{V}\right|-\right\rangle+\left\langle+\left|\mathcal{V} G_{+}: \Omega: G_{-} \mathcal{V} G_{-} \mathcal{V}\right|-\right\rangle \\
& +\left\langle+\left|: \Omega: G_{-} \mathcal{V} G_{-} \mathcal{V} G_{-} \mathcal{V}\right|-\right\rangle, \quad\left(\mathcal{V}=\mathcal{V}_{1}\right) .
\end{aligned}
$$

We evaluate, as an example, $\left\langle+\left|\mathcal{V}_{1} G_{+} \mathcal{V}_{1} G_{+} \mathcal{V}_{1} G_{+}: \Omega:\right|-\right\rangle$. Using the technique described in the previous subsection,

$$
\left\langle+\left|\mathcal{V}_{1} G_{+} \mathcal{V}_{1} G_{+} \mathcal{V}_{1} G_{+}: \Omega:\right|-\right\rangle \rightarrow \frac{3 ! 3 !}{3 ! 3 !}\left[\int_{1_{C} 2_{C}}\left\{\frac{1}{\omega_{+}\left(1_{C}\right)+\omega_{+}\left(2_{C}\right)}\right\}\left\{\frac{1}{\omega_{+}\left(1_{C}\right)+\omega_{+}(q)+2 \omega_{+}\left(2_{C}\right)}\right\}\right.
$$




$$
\begin{aligned}
& \cdot\left\{\frac{1}{2 \omega_{+}\left(1_{C}\right)+2 \omega_{+}\left(2_{C}\right)+\omega_{+}(p)+\omega_{+}(q)}\right\} S_{-}(p) \Gamma_{1}\left(p, 1_{C}\right) T_{+}\left(1_{C}\right) \Gamma_{1}\left(1_{C}, 2_{C}\right) T_{+}\left(2_{C}\right) \\
& \left.\cdot \Gamma_{1}\left(2_{C}, q\right) S_{+}(q)+(5 \text { terms })\right],
\end{aligned}
$$

where we have taken two, four and six particle states in the evaluation of the first, second and third $G_{+}$from the left, respectively. (The contributions of the other states do not lead to the divergent terms. This situation is quite similar to the case of eq. (64), which does not exhibit the infrared divergence for the first term of the Taylor expansion. These terms do not have enough $\cos \beta(p)^{-1}$ factors to develop logarithmic divergences. ) In eq. (76) the six terms are different with each other only in the momentum arguments in $\omega_{+}$. For example, there is a term proportional to,

$$
\begin{aligned}
& \left\{\frac{1}{\omega_{+}(q)+\omega_{+}\left(1_{c}\right)}\right\}\left\{\frac{1}{\omega_{+}(q)+\omega_{+}\left(1_{c}\right)+\omega_{+}(p)+\omega_{+}\left(2_{c}\right)}\right\} \\
& \cdot\left\{\frac{1}{\omega_{+}(q)+2 \omega_{+}\left(1_{c}\right)+\omega_{+}(p)+2 \omega_{+}\left(2_{c}\right)}\right\} .
\end{aligned}
$$

Such differences are irrelevant for the calculation of the divergent parts of the vertex function, as will be seen later. Multiplying eq. (76) with $A_{\mu}(t)$, and performing the path integral over gauge fields, we obtain (for the one-particle irreducible contributions)

$$
\begin{aligned}
& (2 \pi)^{4} \delta_{P}(p+t-q) \sum_{\rho} D_{\mu \rho}(t) e^{-\frac{i}{2}(p+t-q)_{\rho} a}\left\{S_{-}(p) \frac{a}{\lambda} \Gamma_{\rho}(p, q) S_{+}(q)\right\} \\
& \Gamma_{\rho}(p, q)=\frac{\lambda}{a}(i g)^{3} \int_{k}\left\{\frac{1}{\omega_{+}(q-k)+\omega_{+}(p-k)}\right\}\left\{\frac{1}{2 \omega_{+}(q-k)+\omega_{+}(p-k)+\omega_{+}(p)}\right\} \\
& \cdot\left\{\frac{1}{2 \omega_{+}(q-k)+2 \omega_{+}(p-k)+\omega_{+}(p)+\omega_{+}(q)}\right\} \sum_{\nu \tau} V_{1 \nu}(2 p-k) T_{+}(p-k) V_{1 \rho}(p+q-2 k) \\
& \cdot T_{+}(q-k) V_{1 \tau}(2 q-k) D_{\nu \tau}(k)+(5 \text { terms }) .
\end{aligned}
$$

As is seen, these terms are described by the Feynman diagram Fig. 2. (c). In the limit $a \rightarrow 0, \Gamma_{\rho}(p, q)$ is evaluated in the same way to $\Sigma(p)$. First we perform the analytic continuation (54) and the rescaling of the loop momentum $k \rightarrow \bar{k}=k a$. Then we expand $\Gamma_{\rho}(p, q)$ with respect to the external momentums $p$ and $q$ to extract the divergence in the limit $a \rightarrow 0$. In the present case, only the zero term of the Taylor expansion leads to the logarithmic divergence, which appears as the infrared divergence in the $\bar{k}$ integration. The infrared divergence takes place only in the vicinity $\bar{k} \simeq 0$, because of the pole structure of the propagators, and is evaluated by expanding the integrand around $\bar{k}=0$. 
Therefore, all the $\omega_{+}$factors are (after the rescaling) reduced to the constant $\lambda$ in this calculation. Summing up all the six terms, the result of the calculation is,

$$
\begin{aligned}
& \Gamma_{\rho}(p=0, q=0)=\frac{1}{8}(i g)^{3} \frac{1}{2}\left(1-\gamma_{5} T_{c}\right) \frac{1}{16 \pi^{2}} \gamma_{\rho}\{1-(1-\xi)\}\left\{\frac{1}{\varepsilon}+\log (\kappa a)^{2}\right\} \\
& + \text { finite terms. }
\end{aligned}
$$

The finite part is also evaluated in the same way as the self-energy case, and is given by,

$$
\begin{aligned}
\Gamma_{\rho}(p, q)-\Gamma_{\rho}(0,0)= & -\frac{1}{8}(i g)^{3} \frac{1}{2}\left(1-\gamma_{5} T_{c}\right) \frac{1}{16 \pi^{2}} \gamma_{\rho}\{1-(1-\xi)\}\left\{\frac{1}{\varepsilon}+\log (\kappa / \mu)^{2}\right. \\
& +p, q, \mu \text { dependent terms }\}
\end{aligned}
$$

where we introduced the renormalization point $\mu . \Gamma_{\rho}(p, q)$ is the sum of the above two terms and is independent of $\kappa$. Similarly,

$$
\begin{aligned}
& \left\langle+\left|G_{+} \mathcal{V} G_{+} \mathcal{V}: \Omega: G_{-} \mathcal{V}\right|-\right\rangle \rightarrow(2 \pi)^{4} \delta_{P}(p+t-q) \sum_{\rho} D_{\mu \rho}(t) e^{-\frac{i}{2}(p+t-q)_{\rho} a} \\
& \cdot\left\{S_{-}(p) \frac{a}{\lambda} \Gamma_{\rho}^{1}(p, q) S_{+}(q)-S_{+}(p) \frac{a}{\lambda} \Gamma_{\rho}^{2}(p, q) S_{+}(q)-S_{-}(p) \frac{a}{\lambda} \Gamma_{\rho}^{3}(p, q) S_{-}(q)\right\},
\end{aligned}
$$

where $\Gamma_{\rho}^{1,2,3}(p, q)$ are evaluated in the same way and they are given by (in the limit $a \rightarrow 0)$,

$$
\Gamma_{\rho}^{1,2,3}(p, q) \rightarrow \frac{1}{8}(i g)^{3} \frac{1}{2}\left(1-\gamma_{5} T_{c}\right) \frac{1}{16 \pi^{2}} \gamma_{\rho}\{1-(1-\xi)\} \log (a \mu)^{2}+\text { finite terms. }
$$

The other two terms, $\left\langle+\left|\mathcal{V} G_{+}: \Omega: G_{-} \mathcal{V} G_{-} \mathcal{V}\right|-\right\rangle$ and $\left\langle+\left|: \Omega: G_{-} \mathcal{V} G_{-} \mathcal{V} G_{-} \mathcal{V}\right|-\right\rangle$, in eq. (68) are also described by the Feynman diagrams Fig. 2. (c) and yield similar results. Thus, all the divergent terms respect the correct chiral property of the vertex. Summing up all the contributions, the renormalization factor of the vertex is,

$$
\begin{aligned}
Z_{1} & =1+\left\{\frac{1}{8}+\frac{3}{8}+\frac{3}{8}+\frac{1}{8}\right\} \frac{g^{2}}{16 \pi^{2}}\left(\log (a \mu)^{2}+\text { const }\right) \\
& =Z_{2},
\end{aligned}
$$

where $1 / 8,3 / 8,3 / 8$ and $1 / 8$ indicate the contributions of the first, second, third and fourth terms in eq. (75), respectively. This proves that the vertex is correctly renormalized. 


\section{Discussion}

We have confirmed that, taking into account the dynamical nature of the gauge fields, the fermion propagator and fermion-fermion-gauge boson vertex are properly renormalized preserving the correct chiral properties. Our analysis, together with the studies of the vacuum polarization [7] and the gauge boson n-point functions [9], proves the renormalizability of a chiral gauge theory on the lattice in the overlap formulation at one loop level.

Within the one loop level, phase conventions of the states $|A \pm\rangle$, namely the imaginary parts of $\alpha_{ \pm}(A)$ do not affect our calculations. For higher orders, however, the phase conventions of $\alpha_{ \pm}(A)$, which, in some case, might not be consistent with the gauge invariance, will enter into the analysis. Better understanding of this point, even though within the perturbation theory, will provide qualitatively new informations on this formulation.

\section{Acknowledgment}

We would like to thank Y. Kikukawa, R. Narayanan, S. Randjbar-Daemi and G. Thompson for useful discussions, and M. O'Loughlin for useful comments on the manuscript.

\section{References}

[1] D. B. Kaplan, Phys. Lett. B288, (1992), 342.

[2] H. Aoki, S. Iso, J. Nishimura and M. Oshikawa, Modern. Phys. Lett. A9, (1994), 1755; S. Aoki and H. Hirose, Phys. Rev. D49, (1994), 2604; S. Aoki and Y. Kikukawa, Modern. Phys. Lett. A8, (1993), 3517; S. Aoki and K. Nagai, Phys. Rev. D53, (1996), 5058; S. Chandrasekharan, Phys. Rev. D49, (1994), 1980; M. Creutz and I. Horvath, Nucl. Phys. B(Proc. Suppl.) 34, (1994), 583; M. Creutz, M. Tytgat, C. Rebbi and S. Xue, hep-lat/9612017; J. Distler and S-J. Rey, heplat/9305026; V. Furman and Y. Shamir, hep-lat/9405004; M. F. L. Golterman, K. Jansen and D. B. Kaplan, Phys. Lett. B301, (1993), 219; M. F. L. Golterman, K. Jansen, D. N. Petcher and J. Vink, Phys. Rev. D49, (1944), 1606; 
M. F. L. Golterman and Y. Shamir, hep-lat/9409013; A. Hulsebos, C. P. KorthalsAltes and S. Nicolis, hep-th/9406003; K. Jansen, Phys. Lett. B288, (1992), 348; hep-lat/9410108; K. Jansen and M. Schmaltz, Phys. Lett. B296, (1992), 374; D. B. Kaplan, Nucl. Phys. B(Proc. Suppl.)30, (1993), 597; T. Kawano and Y. Kikukawa, Phys. Rev. D50, (1994), 5365; Y. Kikukawa and S. Miyazaki, Prog. Theor. Phys. 96, (1996), 1189; R. Narayanan and H. Neuberger, Phys. Lett. B302, (1993), 62; C. P. Korthals-Altes, S. Nicolis and J. Prades, Phys. Lett. B316, (1993), 339; R. Narayanan, Nucl. Phys. B(Proc. Suppl.) 34, (1994), 95; Y. Shamir, Nucl. Phys. B406, (1993), 90; Nucl. Phys. B417, (1993), 167; Phys. Lett. B305, (357), 1992; Z. Yand, Phys. Lett. B296, (1992), 151.

[3] R. Narayanan and H. Neuberger, Nucl. Phys. B412, (1994), 574; Phys. Rev. Lett. 71, (1993), 3251; Nucl. Phys. B(Proc. Suppl.) 34, (1994), 95, 587; Nucl. Phys. B443, (1995), 305.

[4] Under certain assumptions a no-go theorem was proved by Nielsen and Ninomiya for the regularization of a chiral fermion on a lattice, Nucl. Phys. B185, (1981), 20; Nucl. Phys. B195, (1982), 541; Nucl. Phys. B193, (1981), 173. The domain wall fermion and overlap formulation evade this theorem by implicitly introducing an infinite number of fermions at each lattice point.

[5] Narayanan and Neuberger in Refs. [3]; S. Randjbar-Daemi, private communication.

[6] Narayanan and Neuberger in Refs. [3]; S. Randjbar-Daemi and J. Strathdee, Phys. Lett. B348, (1995), 543; Phys. Rev. D51, (1995), 6619; Nucl. Phys. B443, (1995), 386; Phys. Lett. B402, (1997), 134.

[7] S. Randjbar-Daemi and J. Strathdee, Nucl. Phys. B461, (1996), 305; Y. Kikukawa, Nucl. Phys. B(Proc. Suppl.) 47, (1996), 599.

[8] S. Aoki and R. B. Levien, Phys. Rev. D51, (1995), 3790.

[9] S. Randjbar-Daemi and J. Strathdee, Nucl. Phys. B466, (1996), 335.

[10] Y. Kikukawa, hep-lat/9705024; hep-lat/9707010. 
[11] A. Yamada, hep-lat/9705040.

[12] R. Narayanan and H. Neuberger, Phys. Lett. B348, (1995), 549; Nucl. Phys. B477, (1996), 521; R. Narayanan, H. Neuberger and P. Vranas, Phys. Lett. B353, (1995), 507; Y. Kikukawa, R. Narayanan and H. Neuberger, Phys. Lett. B399, (1997), 105; hep-lat/9705006; S. Aoki, K. Nagai and S. V. Zenkin, hep-lat9705001.

[13] J. Nishimura, hep-lat/9701013; J. Nishimura and N. Maru, hep-th/9705152; R. Narayanan and J. Nishimura, hep-th/9703109; H. Neuberger, hep-lat/9707022; Y. Kikukawa and H. Neuberger, hep-lat/9707016.

[14] The Euclidean gamma matrices $\gamma_{\mu}^{E}$ are related to the Minkowskian gamma matrices $\gamma_{M}^{\mu}$ as $\gamma_{i}^{E}=-i \gamma_{M}^{i}$ (for $\left.i=1,2,3\right)$ and $\gamma_{4}^{E}=\gamma_{M}^{0}$.

[15] Nontrivial phases of $\alpha_{ \pm}(A)$ appear at the order $\mathcal{O}\left(g^{3}\right)$ [9, 10] and do not affect the calculations of the propagator and vertex at one loop level.

[16] H. Kawai, R. Nakayama and K. Seo, Nucl. Phys. B189, (1981), 40.

[17] N. Kawamoto, Nucl. Phys. B190[FS3], (1981), 617.

[18] L. H. Karsten and J. Smit, Nucl. Phys. B183, (1981), 103.

[19] To be specific, we understand that the regularization (54) is performed after the manipulation of the gamma matrices in $\Sigma(p)$ even though we introduce from the beginning before the calculation of the gamma matrices. In this sense, the regularization (54) is similar to the $\overline{D R}$ regularization of the ultraviolet divergence.

\section{Figure Caption}

Fig. 1 The Feynman diagrams describing the self-energy corrections. The diagram (a) yields the linearly and logarithmically divergent terms, while the diagram (b) yields only the linearly divergent terms. The linearly divergent terms in these diagrams are reduced to finite wave function renormalization factors at the level of the propagator. 
Fig. 2 The Feynman diagrams describing the vertex corrections. The diagrams (a) and (b) lead only to the finite corrections. The diagram (c), containing only the interaction $\mathcal{V}_{1}$, leads to the logarithmic divergences.

\section{Figures}

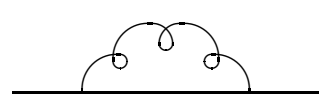

Fig. 1. (a).
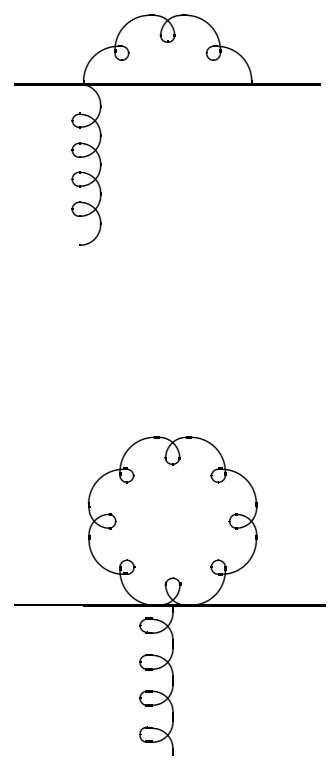

Fig. 2. (b).

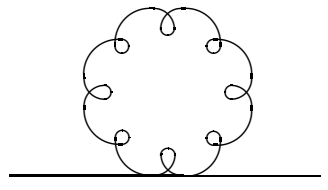

Fig. 1. (b).

Figs. 2. (a).
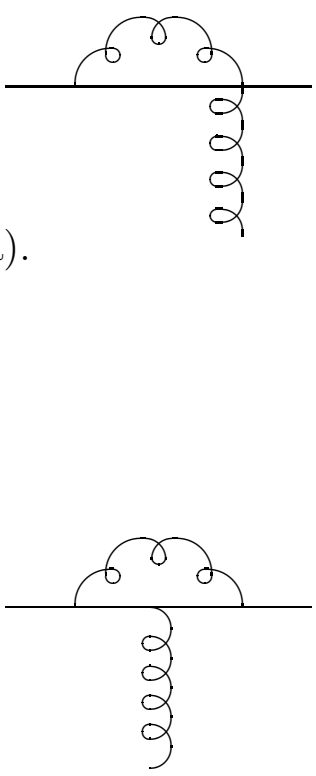

Fig. 2. (c). 